\title{
NATURAL CONVECTION IN HIGH ASPECT RATIO THREE- DIMENSIONAL ENCLOSURES WITH UNIFORM HEAT FLUX ON THE HEATED WALL
}

\author{
T. Dias Jr., \\ and L. F. Milanez \\ Universidade Estadual de Campinas \\ Faculdade de Engenharia Mecânica \\ Departamento de Energia \\ 13081-970 \\ Campinas - São Paulo \\ Brasil \\ titodiasjr@uol.com.br

\begin{abstract}
In this work, the laminar natural convection in high aspect ratio three-dimensional enclosures has been numerically studied. The enclosures studied here were heated with uniform heat flux on a vertical wall and cooled at constant temperature on the opposite wall. The remaining walls were considered adiabatic. Fluid properties were assumed constant except for the density change with temperature on the buoyancy term. The governing equations were solved using the finite volumes method and the dimensionless form of these equations has the Prandtl number and the modified Rayleigh number as parameters. The influences of the Rayleigh number and of the cavity aspect ratio on the Nusselt number, for a Prandtl number of 0.7, were analyzed. Results were obtained for values of the modified Rayleigh number up to $10^{6}$ and for aspect ratios ranging from 1 to 20. The results were compared with two-dimensional results available in the literature and the variation of the average Nusselt number with the parameters studied were discussed.

Key words: natural convection, numerical study.
\end{abstract}

\section{INTRODUCTION}

Natural convection heat transfer has increasingly attracting the interest of researchers. This is due to its importance on many engineering applications, such as solar collectors, environmental engineering and electronic packaging. Many studies considering natural convection on plates, channels and enclosures with heated walls have been performed (Ganzarolli and Milanez, 1995). De Vahl Davis (1983) studied numerically a two-dimensional square cavity with isothermal walls, and the results are frequently used as a benchmark to validate new simulation softwares. Another example is Oosthuizen (2000), who studied numerically a twodimensional enclosure with uniform heat flux on a vertical wall and constant temperature on the other vertical wall while the horizontal walls were considered adiabatics.

However, two-dimensional models are still limited compared to the complexity of many real situations, where three-dimensional effects are frequently present. Some of these effects have been included in the work of Jaluria (1976), who considered an axisymmetric plume flow over hemispherical sources, and in the paper of Kurdyumov and Liñán (1999), who studied flow over spherical sources.

Oosthuizen and Paul (1998) studied a horizontal three-dimensional cavity heated from below and cooled from above with uniform temperatures. They analyzed the aspect ratio influence on the critical Rayleigh number, the value of this parameter where the conductive regime becomes a convective regime.

Tric et al. (2000) obtained accurate results, with the pseudo-spectral Chebyshev algorithm based on the projection-diffusion approach, for a vertical cubical cavity with isothermal opposite walls. According to them, the global relative error of the results is in the range of $0.03-0.05 \%$ for Rayleigh number varying from $10^{3}$ to $10^{7}$.

In this work we studied three-dimensional enclosures and compared the results with those available for two-dimensional geometries. In order to simulate a geometric configuration used frequently in two-dimensional experimental approximation, we considered three-dimensional enclosures with uniform heat flux on a vertical wall and the opposite one cooled with uniform temperature. The remaining walls were considered adiabatic. According to Oosthuizen (2000), the enclosure width $(W)$ was taken as the characteristic length, air was the working fluid $(P r=0.7)$ and the depth aspect ratio $\left(A_{x}\right)$ was taken as equal to the aspect ratio $A_{z}$. The study was carried out by varying $A_{z}$ from 1 to 20 and the modified Rayleigh number from $10^{3}$ to $10^{6}$.

\section{PROBLEM FORMULATION}

Laminar flow and constant fluid properties 


\section{TECNOLOGIA/TECHNOLOGY}

except for the density change with temperature on the buoyancy term were assumed. The finite volume control method was used for the discretization of the elliptic governing equations, the steady three-dimensional versions of continuity, momentum and energy equations (Dias-Jr, 2003). Following Oosthuizen (2000), the dimensionless parameters considered have been defined as below.

\section{Dimensionless Temperature}

$$
\theta=\left(T-T_{c}\right) /(q W / k)
$$

where $T c$ is the temperature of the cooled wall, $q$ is the heat flux, $k$ is the thermal conductivity and $W$ is the enclosure width.

\section{Aspect Ratio Az}

$$
A_{z}=H / W
$$

where $H$ is the enclosure height.

\section{Modified Rayleigh Number}

$$
R a_{q}=\mathrm{b} g q W^{4} / \mathrm{kna}
$$

where $b$ is thermal expansion coefficient, $a$ is the thermal diffusivity and $\mathrm{n}$ is the kinematic viscosity.

\section{Average Nusselt Number}

$$
N u=\frac{q W}{k\left(\bar{T}_{h}-T_{c}\right)}=\frac{1}{\overline{\mathrm{q}}_{h}}
$$

where $\bar{T}_{h}$ is the mean temperature over the heated wall and $\bar{\theta}_{h}$ is the mean dimensionless temperature.

The boundary conditions have been established to simulate a geometric configuration used frequently in two-dimensional experimental approximation. Three-dimensional enclosures were considered with uniform heat flux on a vertical wall and the opposite one cooled with uniform temperature. The remaining walls were considered adiabatic.

\section{NUMERICAL PROCEDURE}

The numerical solution was obtained using the SIMPLE algorithm, since the flow was assumed incompressible. The interpolation of gradients of velocity and temperature used the QUICK algorithm, implemented on Fluent 5.4 software package.

The resulting algebraic system was solved by the point-to-point Gauss-Seidel algorithm, which is known to have a slow convergence. To accelerate this convergence the algebraic multigrid method was used.

The governing equations were solved in the dimensional form and then the results were changed to the dimensionless form. The fluid properties were taken from Bejan (1994).

\section{RESULTS AND DISCUSSION}

The influence of the aspect ratio $A_{z}$ on the heat transfer rate in vertical three-dimensional enclosures has been obtained and compared to twodimensional results obtained by Oosthuizen (2000).

Figure 1 shows one of the results obtained by Oosthuizen (2000). It is possible to observe the average Nusselt number variation with the aspect ratio $A_{z}$ and the modified Rayleigh number, and a comparison between the two and three-dimensional cases. It can be seen that the average Nusselt number increases with the modified Rayleigh number for both cases and the difference between these cases increases with the modified Rayleigh number as the aspect ratio $A_{z}$ increases. Therefore the two-dimensional configuration frequently used deviates from the experimental results obtained for three-dimensional geometry as the modified Rayleigh number increases.

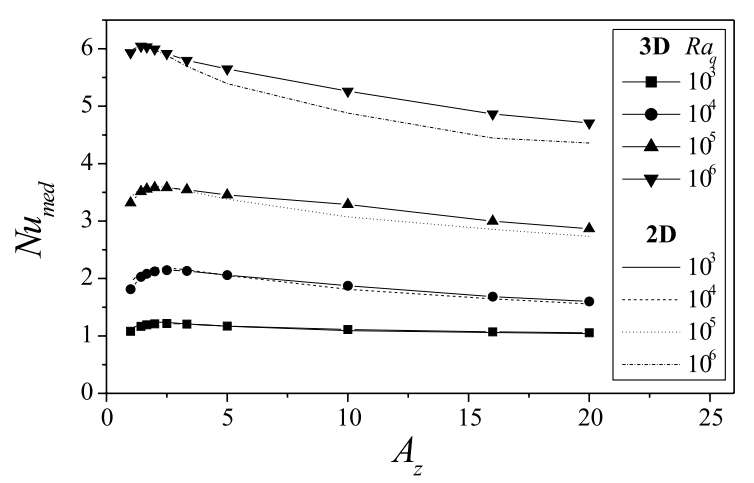

Figure 1. Average Nusselt number variation with aspect ratio $A_{z}$. 
It can also be noticed a point where the average Nusselt number is a maximum, and this point and the corresponding average Nusselt number change with the modified Rayleigh number too. This has been pointed out by Oosthuizen (2000), who derived an average Nusselt number function depending only on the aspect ratio $A_{z}$. This curve was obtained by dividing each $N u$ x $A_{z}$ by their corresponding $N u_{\max } \times A_{z \max }$, resulting in a function independent of the modified Rayleigh number.

Independently of the numerical results, it is important to analyze the dependence between the dimensionless parameters used. As previously defined by Eq. (4), the average Nusselt number is inversely proportional to the dimensionless mean temperature of the heated wall. And the dimensionless temperature is inversely proportional to the heat flux $(q)$ and to the enclosure width $(W)$; thus, increasing the modified Rayleigh number, by increasing $q$ or $W$, the dimensionless temperature decreases and the average Nusselt number increases. Although the Oosthuizen's study is based on the average Nusselt number dependence on the modified Rayleigh number and on the aspect ratio $A_{z}$, these parameters are not independent variables.

Considering only the influence of the modified Rayleigh number, it increases with the heat flux, leading to a decreasing of the dimensionless temperature, since the heat flux has been used in the temperature dimensionless approach. To eliminate this dependence we have analyzed the relation between the average Nusselt number and the modified Rayleigh number, as shown in Fig. 2. It can be seen the curves almost coinciding in the plot.

Considering now only the aspect ratio influence, since this is a ratio between the height and the width of the enclosure, its increasing is equivalent to a decreasing of the characteristic length $(W)$, and this results in an increasing of the dimensionless temperature and consequent average Nusselt number decreasing. A decreasing of $W$ leads also to a decreasing in the modified Rayleigh number, since this number depends on $W^{4}$, this meaning that $R a_{q}$ is not independent of the aspect ratio. Therefore, to eliminate the aspect ratio influence we have divided the average Nusselt number by the aspect ratio to the third power and by the modified Rayleigh number, as shown in Fig. 3. It can be noticed that the point of maximum has disappeared revealing the real behavior of the curve. This is important since this point of maximum could lead the reader to look for a change on the flow which could justify this point, an artifact arisen from the dimensionless approach.

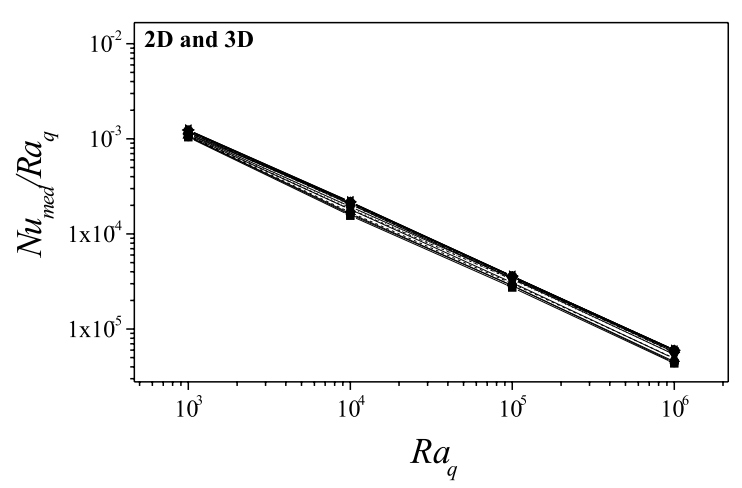

Figure 2. Variation of average Nusselt number normalized by the modified Rayleigh number as a function of the modified Rayleigh number

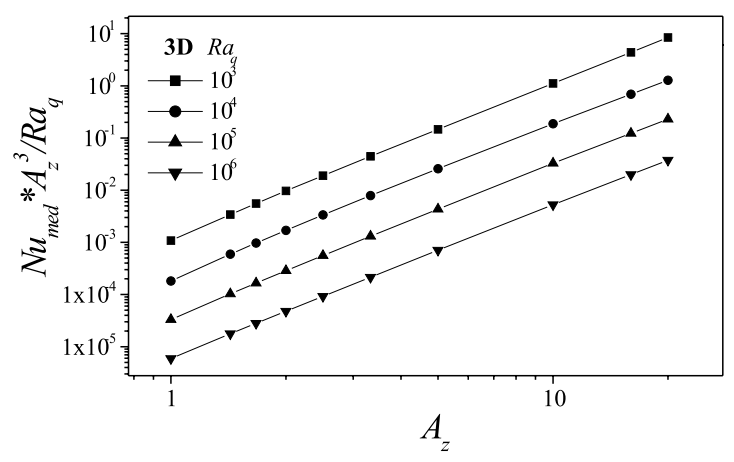

Figure 3. Normalized average Nusselt number variation with aspect ratio for the three-dimensional case.

\section{CONCLUSIONS}

The results obtained in this work indicate that the two-dimensional approximation, frequently compared to experimental results, deviates from the three-dimensional results as the modified Rayleigh number increases.

It was shown that the point of maximum, pointed out by Oosthuizen (2000), has disappeared with a normalization process revealing the real behavior of the average Nusselt number with the aspect ratio $A_{z}$ and with the modified Rayleigh number. This is important since that point of maximum could lead the reader to look for a change on the flow which could justify this point, an artifact arisen from the dimensionless approach. 


\section{ACKNOWLEDGEMENTS}

This work was supported by FAPESP (99/ 12588-3).

\section{REFERENCES}

Bejan, A., 1994, Convection Heat Transfer, 2nd ed., John Wiley \& Sons, New York, 656p.

De Vahl Davis, G., 1983, Natural Convection in a Square Cavity: a Benchmark Numerical Solution, International Journal of Numerical Methods in Fluids, Vol. 3, pp.249-264.

Dias-Jr, T. Convecção Natural Tridimensional Sobre Fontes de Calor Discretas. Tese (Doutorado) - Faculdade de Engenharia Mecânica, Universidade de Campinas, 2003. (in Portuguese)

Ganzarolli, M. M. and Milanez, L. F., 1995, Natural Convection in Rectangular Enclosures Heated from Below and Symmetrically Cooled from the Sides, International Journal of Heat and Mass Transfer, Vol. 38, No. 6, pp. 1063-1073.

Jaluria, Y., 1976, Natural Convection Flow Interaction Above a Heated Body, Letters in Heat and Mass Transfer, Vol. 3, pp. 457-466.

Kurdyumov, V. N. and Liñán, A., 1999, Free Convection from a Point Source of Heat, and Heat Transfer from Spheres at Small Grashof Numbers, International Journal of Heat and Mass Transfer, Vol. 42, pp. 3849-3860.

Oosthuizen, P. H. and Paul, J. T., 1998, A Numerical Study of Three-dimensional Natural Convection in a Horizontal Enclosure with a Uniform Heat Flux on the Lower Surface, Proceedings of the $11^{\text {th }}$ International Heat Transfer Conference, Vol. 3, pp. 391-396.

Oosthuizen, P. H., 2000, Natural Convective Flow in a High Aspect Ratio Rectangular Enclosure with a Uniform Heat Flux on the Heated Wall, Proceedings of the 3rd European Thermal Sciences Conference, Vol. 1, pp. 159-164.

Tric, E., Lambrosse, G. and Betrouni, M., 2000, A First Incursion into the 3D Structure of Natural Convection on air in a Differentially Heated Cubic Cavity, from Accurate Numerical Solutions, International Journal of Heat and Mass Transfer, Vol. 43, pp. 4043-4056. 